\title{
USO DEL MODELO IRAP PARA EVALUAR LA SEGURIDAD VIAL EN CARRETERAS DE DOS CARRILES EN ECUADOR
}

\author{
Using the iRAP model to assess road safety on two-lane \\ roads in Ecuador
}

\author{
Yasmany García Ramírez ${ }^{\mathrm{a}}$, Jandry CAmacho $^{\mathrm{b}}$ y Jefferson Montoya ${ }^{\mathrm{c}}$ \\ Recibido: 15/05/2021 • Aprobado: 18/05/2021
}

Cómo citar: García-Ramírez, Y., Camacho, J., \& Montoya, J. (2021). Uso del modelo iRAP para evaluar la seguridad vial en carreteras de dos carriles en Ecuador. Ciencia, Ingenierías y Aplicaciones, 4(1), 7-23. DOI: https://doi.org/10.22206/cyap.2021.v4i1.pp7-23

\section{Resumen}

El International Road Assessment Programme (iRAP) permite evaluar la seguridad vial que ofrecen tramos de carreteras. El modelo busca reducir el número de siniestros de tránsito y la gravedad de los mismos, ofreciendo información valiosa para quienes investigan o toman decisiones en las vías. En Ecuador, a pesar de tener un alto número de siniestros viales, solo se suelen hacer inspecciones de seguridad vial esporádicas, que muy pocas veces son aplicadas por falta de conocimiento técnico. Este artículo tiene como objetivo explorar el uso del iRAP mediante su aplicación en una vía de dos carriles en dos carreteras en Ecuador. Para ello, se analizó a profundidad el modelo iRAP en dos tramos de carreteras de dos carriles, analizando un total de $25 \mathrm{~km}$. En esos tramos se recolectó información principalmente con el equipo Video VBOX Lite. Los resultados muestran que, a pesar de tener un alto número de variables, es posible aplicar el iRAP a carreteras de dos carriles. También se muestra que el modelo tiene dos variables que no están claramente descritas en los documentos del iRAP y se encontraron algunos errores menores en el ejemplo disponible en la web. A pesar de esto, se pudo determinar el puntaje de calificación por estrellas (SRS) y calificación por

\footnotetext{
a Profesor Adjunto, Departamento de Ingeniería Civil, Universidad Técnica Particular de Loja, San Cayetano Alto, Loja, Ecuador Correo-e: ydgarcia1@utpl.edu.ec

b Estudiante de pre-grado, Departamento de Ingeniería Civil, Universidad Técnica Particular de Loja. Correo-e: jrcamacho2@utpl.edu.ec

c Estudiante de pre-grado, Departamento de Ingeniería Civil, Universidad Técnica Particular de Loja. Correo-e: jdmontoya4@utpl.edu.ec
} 
estrellas (SR). La mayoría de los tramos tuvieron una estrella y con la aplicación de las contramedidas seleccionadas se alcanzó hasta dos estrellas. Este estudio se puede reproducir fácilmente a otras carreteras del pais o en otros países.

Palabras clave: Ecuador; Ingeniería Vial; seguridad del transporte; carretera; política del transporte

\begin{abstract}
The International Road Assessment Program (iRAP) makes it possible to assess road safety in the road sections. The model seeks to reduce the traffic accidents and their severity, offering valuable information for researchers or politicians that decided on road development. In Ecuador, despite having a high number of road accidents, only sporadic road safety inspections are usually carried out. These inspections are rarely applied due to a lack of technical knowledge. This article aims to explore the use of iRAP in the Ecuadorian context through its application on a two-lane road in two roads in Ecuador. For this, the iRAP model was analyzed in-depth in two sections of two-lane roads, using a total of $25 \mathrm{~km}$. In these sections, data was collected mainly with the Video VBOX Lite equipment. The results show that despite having a high number of variables, it is possible to apply iRAP to two-lane roads. Also, it shows the model has two variables that are not clearly described in the iRAP documents. So, some minor errors were found in the example available on the web. Despite this, the Star Rating Scores (SRS) and Star Rating (SR) could be determined. Most of the sections had 1 star, and with the application of the selected countermeasures, up to two stars were achieved. This study can be easily reproduced on other roads in the country or other countries.
\end{abstract}

Keywords: Ecuador; Road engineering; transport safety; road; Transport policy 


\section{Introducción}

El International Road Assessment Programme (iRAP) empezó con la década de la seguridad vial 2011-2021 propuesta por las Organización de las Naciones Unidas (ONU, 2011), debido a las preocupantes estadísticas de siniestralidad asociadas a las vías de transporte terrestre (Organización Mundial de la Salud, 2015). El iRAP tiene como objetivo principal reducir la mortalidad en las vías, analizando las relaciones de causa-efecto entre la infraestructura-entorno con los usuarios viales, y presentando valoraciones que representan el nivel de peligrosidad del sitio (Mcinerney \& Smith, 2009).

El iRAP utiliza la calificación por estrellas (SR) para cuantificar el nivel de seguridad vial que ofrece un escenario vial para los diferentes usuarios viales: ocupante del vehículo, motociclista, peatón y ciclista, tal como se muestra en la tabla 1. Esta calificación se basa en un puntaje de la calificación por estrellas (SRS), que se calcula considerando los atributos de la carretera que afectan la seguridad vial y que, posteriormente, se utilizan para diseñar planes de inversión para carreteras más seguras. El iRAP brinda capacitaciones a nivel mundial para promover el uso de su propuesta (iRAP, 2020). A pesar de estos esfuerzos y de investigadores independientes, solo algunos países o regiones han adoptado este programa: Europa, Australia, Estados Unidos de América, Nueva Zelanda, China, India, Brasil, Sudáfrica, Tailandia, Malasia y Pakistán (iRAP, 2006).

Tabla 1. Banda de clasificación de estrellas del modelo iRAP

\begin{tabular}{|c|c|c|c|c|c|}
\hline \multirow{3}{*}{$\begin{array}{l}\text { Calificación } \\
\text { de estrellas } \\
\text { (SR) }\end{array}$} & \multicolumn{5}{|c|}{ Valoración de la calificación de estrellas (SRS) } \\
\hline & \multirow{2}{*}{$\begin{array}{l}\text { Ocupantes } \\
\text { de vehículo y } \\
\text { motociclistas }\end{array}$} & \multirow{2}{*}{ Ciclistas } & \multicolumn{3}{|c|}{ Peatones } \\
\hline & & & Total & A lo largo & Cruzando \\
\hline 5 & $0 \mathrm{a}<2,5$ & $0 \mathrm{a}<5$ & $0 a<5$ & $0 \mathrm{a}<0,2$ & $0 \mathrm{a}<4,8$ \\
\hline 4 & $2,5 a<5,0$ & $5 a<10$ & $5 a<15$ & $0,2 \mathrm{a}<1,0$ & $4,8 \mathrm{a}<14,0$ \\
\hline 3 & $5,0 \mathrm{a}<12,5$ & $10 a<30$ & $15 a<40$ & $1,0 a<7,5$ & $14,0 \mathrm{a}<32,5$ \\
\hline 2 & $12,5 \mathrm{a}<22,5$ & $30 a<60$ & $40 a<90$ & $7,5 \mathrm{a}<15,0$ & $32,5 a<75$ \\
\hline 1 & $\geq 22,5$ & $\geq 60$ & $\geq 90$ & $\geq 15,0$ & $\geq 75$ \\
\hline
\end{tabular}

Fuente: iRAP, 2006 
En América Latina, solo Brasil ha adoptado el modelo, lo cual es preocupante dado el creciente número de siniestros viales en la región (Organización Panamericana de la Salud, 2016). Por ejemplo, Ecuador reporta más de 2000 muertes por esta misma causa (Agencia Nacional de Tránsito, 2020), por lo que se ubica en los primeros lugares de siniestralidad vial en América Latina. En Ecuador, como en otros países en vías de desarrollo, el limitado presupuesto para las obras viales, junto con la falta de técnicos capacitados, hace que las instituciones solo se encarguen, principalmente, del mantenimiento de las vías (prevención, intervenciones en sitios de concentración de siniestros, etc.) pero no de la seguridad vial de las mismas. A pesar de realizarse inspecciones viales, estas tienen una aplicación limitada debido a esa falta de personal calificado.

Todas estas limitaciones han hecho que se deje de lado la aplicación e investigación relacionada con el iRAP en este país. Esto es en contraste a otros países en donde sí se han realizado investigaciones, como por ejemplo el Reino Unido (Jameel \& Evdorides, 2016), Australia (Jurewicz \& Excel, 2016), China (Zhang, Tang, Smith \& Wu, 2014), Canadá (MacAngus et al., 2011), Bangladesh (Hoque, Smith, Hossain \& Mahmud, 2010), Moldavia (Lawson, Barlow, Poran, Petrosyan \& Sevrovic, 2017), entre otros. Estas investigaciones fundamentalmente se han enfocado en ajustar los valores de frecuencia y gravedad de los siniestros viales que afectan el cálculo de los SR.

Este estudio tiene por objetivo evaluar el método iRAP aplicado al análisis de la seguridad de las carreteras de dos carriles del Ecuador. El iRAP es un modelo desarrollado en otros contextos viales y geográficos, por lo que este análisis permitirá conocer si con base en la información disponible y la que se recolecte se puede adoptar el modelo. Además, permitirá explorar las más de 60 variables que se utilizan para carreteras rurales para entender la codificación que hace el modelo y establecer posibles líneas de investigación. Los resultados de esta investigación permitirán que los ingenieros y técnicos, e incluso estudiantes, mejoren su conocimiento en seguridad vial y en las medidas que se puede hacer 
para reducir las probabilidades de fallecimiento en las vías. Para desarrollar el estudio, en primer lugar, se hace una descripción metodológica del experimento, para luego explicar los resultados y resaltar las principales conclusiones.

\section{Métodos}

Para analizar la implementación del iRAP en carreteras rurales, en primer lugar, se hizo una revisión exhaustiva del modelo iRAP. Posteriormente, se eligieron los sitios en donde se aplicaría la metodología, para luego recolectar datos necesarios. Con esos datos, se realizó el procesamiento de los mismos y finalmente se calificó cada uno de los sitios y, de ser el caso, se propusieron contramedidas.

\subsection{Análisis del modelo iRAP}

Una de las primeras actividades fue analizar el modelo iRAP con el objetivo de conocer cada una de las variables y de los factores que intervienen en este procedimiento. Se analizaron los fact sheets disponibles en la página web del iRAP (https://irap.org). Principalmente, se analizó el ejemplo resuelto \#9 (iRAP, 2013), para validar cada variable y/o factor. Esta información también fue revisada en la versión web (https://vida.irap.org/es) para calificar los sitios utilizando el modelo iRAP, denominada ViDA (ChinaRAP, 2020).

\subsection{Selección de los sitios}

Luego de que se entendió el funcionamiento del modelo iRAP, se seleccionaron los sitios para aplicarlo. Se eligieron tramos de carreteras de dos carriles de la provincia de Loja y Zamora Chinchipe (Ecuador): $10 \mathrm{~km}$ en la vía Loja-Yangana y $15 \mathrm{~km}$ en la vía Loja-Zamora, tal como se muestra en la figura 1. Estas carreteras se encuentran ubicadas en topografía montańosa de la cordillera de los Andes. La velocidad máxima legal en estas carreteras es de $90 \mathrm{~km} / \mathrm{h}$. Considerando su importancia para la región, las carreteras son de competencia del gobierno nacional. 


\subsection{Recolección de datos}

Con los tramos de carreteras definidos se elaboró un plan de recolección de datos. En primer lugar, dado que se requiere de algunos elementos del diseño geométrico (curvatura y pendiente), se recolectó información con el equipo Video VBOX Lite. Este equipo tiene instalada una cámara, orientada hacia delante del vehículo, la cual graba un video geo-referenciado. Con la planimetría y altimetría de este recorrido, se obtuvieron datos aproximados del radio de curvatura y de la pendiente longitudinal. Por otro lado, también fue necesario recolectar información del flujo vehicular, peatonal, de motocicletas y bicicletas. Para ello, el flujo vehicular se obtuvo del Observatorio de Seguridad Vial de al UTPL (Observatorio de Seguridad Vial, 2020), mientras que el resto de flujos se obtuvo mediante el conteo en el sitio de interés. Otra variable de alto impacto en la calificación iRAP es la velocidad de operación, la cual se consiguió con ecuaciones calibradas en este tipo de carreteras (García-Ramírez \& Alverca, 2019), aunque también se pudieron obtener en mediciones en campo. El resto de mediciones como, por ejemplo, el ancho del carril, se realizó en el sitio, mientras que otras, como costo de modernización, fueron asumidas con base en los datos recolectados.
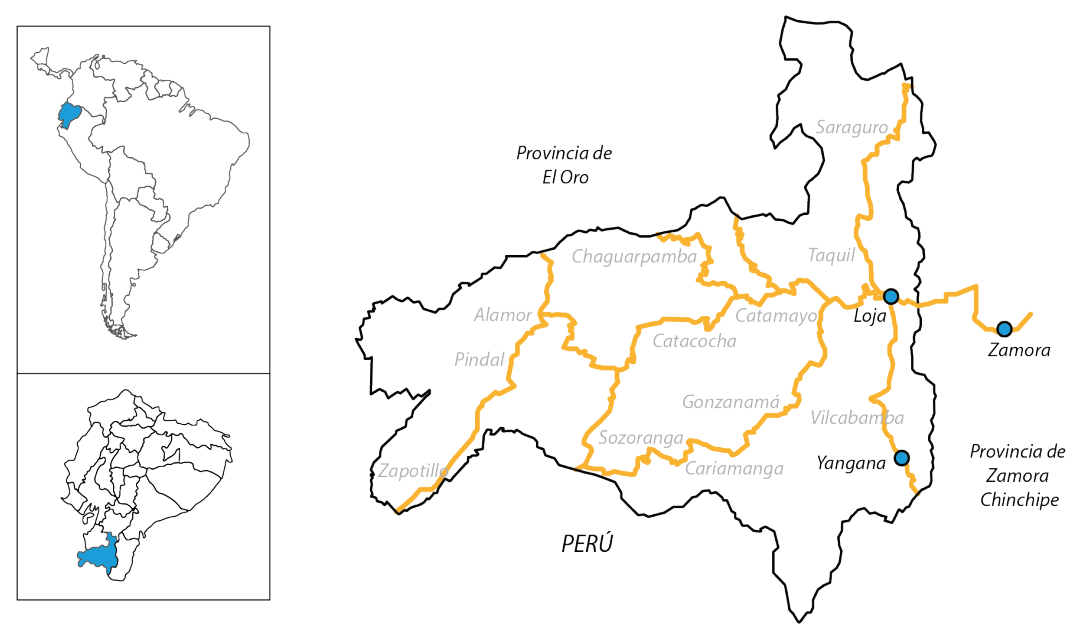

Figura 1. Mapa de las rutas analizadas 


\subsection{Procesamiento de datos}

Luego de la recolección de datos, se procedió a determinar el radio de la curva horizontal y pendiente longitudinal. Para la vía Loja-Yangana se encontraron radios entre 36 y $118 \mathrm{~m}$, mientras que, para la vía Loja-Zamora los radios estuvieron entre 31 y 273 m. Así mismo, las pendientes en la primera vía estuvieron entre $-8,7 \%$ a $10,0 \%$ y para la segunda entre $-8,5 \%$ a 9,6\%. En lo que se refiere a la velocidad de operación, la carretera Loja - Zamora tuvo velocidades entre 50 y 85 $\mathrm{km} / \mathrm{h}$, mientras que, la carretera Loja - Yangana entre 35 y $80 \mathrm{~km} / \mathrm{h}$. En lo que respecta al flujo, la tabla 2 muestra los flujos viales de los tramos analizados.

Tabla 2. Flujos para los diversos usuarios viales que se estimaron para el 2021 de las dos vías en estudio

\begin{tabular}{ccccc}
\hline \multirow{2}{*}{$\begin{array}{c}\text { Tipo de flujo en } \\
\text { el año 2021 }\end{array}$} & \multicolumn{2}{c}{ Vía Loja - Zamora } & \multicolumn{2}{c}{ Vía Loja -Yangana } \\
\cline { 2 - 5 } & $\begin{array}{c}\text { Flujo en tramo } \\
\text { suburbano }\end{array}$ & $\begin{array}{c}\text { Flujo en } \\
\text { tramo rural }\end{array}$ & $\begin{array}{c}\text { Flujo en tramo } \\
\text { suburbano }\end{array}$ & $\begin{array}{c}\text { Flujo en } \\
\text { tramo rural }\end{array}$ \\
\hline Vehicular & 2548 & 2548 & 4160 & 4160 \\
\hline Peatonal total $^{* *}$ & 31 & 0 & 0 & 0 \\
\hline Bicicletas* $^{\text {Motocicletas* }}$ & 1 & 0 & 0 & 0 \\
\hline Myyyyy$^{*}$ & 4 & 0 & 0 & 0 \\
\hline
\end{tabular}

* En hora pico a lo largo de la carretera, ${ }^{* *}$ En hora pico que cruzan más a lo largo de la carretera.

\subsection{Calificación de los tramos, suavización y contramedidas}

Cada uno de los tramos analizados se dividieron en sub-tramos o secciones de $100 \mathrm{~m}$ tal como lo menciona el procedimiento iRAP. Con esa división se obtuvieron 100 sub-tramos en la vía Loja - Yangana y 150 subtramos en la vía Loja - Zamora. Cada uno de los valores de los atributos de cada subtramo fueron ingresados al programa en línea ViDA (https://vida.irap.org/es). El resultado fue el puntaje de calificación por estrellas (SRS) para cada usuario vial. Los datos SRS fueron dibujados a lo largo de la carretera y se suavizaron para obtener el número de estrellas. 
El iRAP propone una suavización por secciones homogéneas y por longitud, no obstante, dado que ninguna de las dos se ajustaba al tipo de resultado gráfico que se quería mostrar, se decidió utilizar la suavización doble (media móvil de 9 como ancho de ventana + exponencial con $\alpha=0,1)$. A manera de ejemplo, se muestra en la figura 2 los valores SRS para el ocupante del vehículo en la vía Loja - Yangana. En esta figura se observa el valor SRS sin suavizar el SRS suavizado. Con base en este valor y los umbrales de cada estrella se calcularon el número de estrellas (SR). Finalmente, de las 94 contramedidas se eligieron algunas de ellas y se verificó su modificación en el SRS y SR.

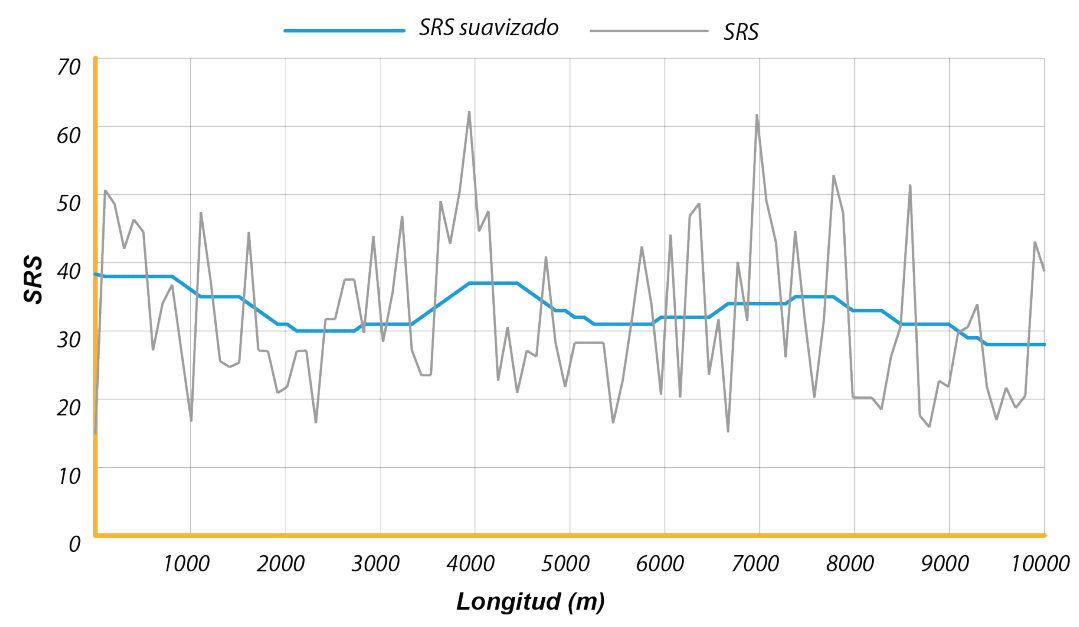

Figura 2. Ejemplo de suavizado para ocupante del vehículo en la vía Loja - Yangana

\section{Resultados}

\subsection{Análisis del modelo iRAP}

Luego de ingresar la informacion del ejercicio resuelto \#9 del IRAP en el programa ViDA se obtuvieron los valores SRS y se compararon con los del ejercicio. En este caso, se encontraron pequeñas diferencias en la selección de algunos factores de los atributos, pero no afectó la valoración final del sitio. Luego de ello, se elaboró una hoja electrónica para entender el proceso de cálculo del programa y modelo. Si bien el 
proceso no es complejo, sí demanda de mucha atención al momento de ingresar esa gran cantidad de variables. Con base en este análisis se detectaron dos elementos que pueden ser una gran fuente de variación: el flujo vehicular y la velocidad de operación. Es decir, el fact sheet del flujo no es claro y no se conoce qué proceso se realiza para obtener los factores. Asimismo, el fact sheet de velocidad, a pesar de que se tiene una figura de donde se pueden obtener los factores, la mayoría de estos factores difieren con los que se obtienen con el software ViDA. Para este estudio se utilizó directamente el software ViDA, por lo que no tuvo que resolverse esos inconvenientes con los temas del fact sheets.

\subsection{Calificación de estrellas}

\subsubsection{Via Loja-Zamora}

Los resultados del modelo iRAP para ocupante adulto se ven en la figura 3 para la carretera Loja - Zamora. En esta figura se han colocado bandas de color para mostrar los umbrales para el cálculo del número de estrellas, tal como se mostró en la tabla 1 .

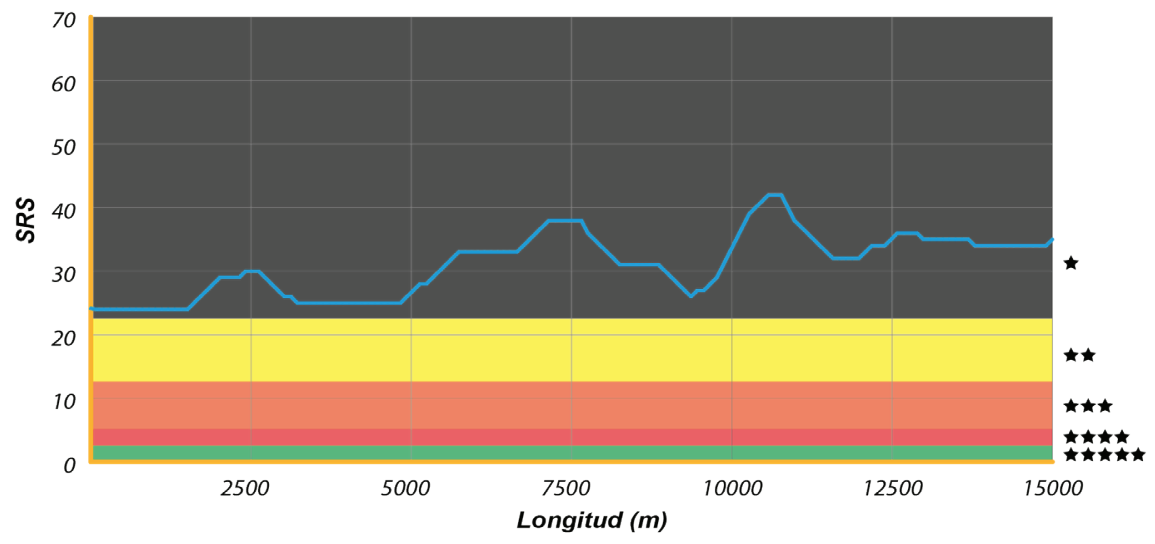

Figura 3. Ocupante de vehículo: valoración de la calificación de estrellas (SRS suavizado) con el número de estrellas (SR) a lo largo de la carretera Loja - Zamora 
En la carretera de la figura 3 se puede ver que todo el tramo tiene sitios con 1 estrella, que corresponden a los escenarios más peligrosos. El iRAP sugiere que, por lo menos los sitios tengan un número de estrellas igual o superior a 3, lo cual se puede realizar utilizando alguna contramedida o combinación de ellas. Por otro lado, en esta vía, existe un subtramo suburbano con presencia de motociclistas, ciclistas y peatones, cuyo resultado de SRS se muestra en la figura 4. En este caso, para motociclistas el riesgo es alto ( 1 estrella), para ciclistas es menor ( 2 estrellas) y para peatones se tienen entre 2 y 3 estrellas. En todos los casos, se pueden hacer mejoras para reducir la probabilidad de fallecimiento o lesión grave en sinestros viales que puedan ocurrir en este subtramo.
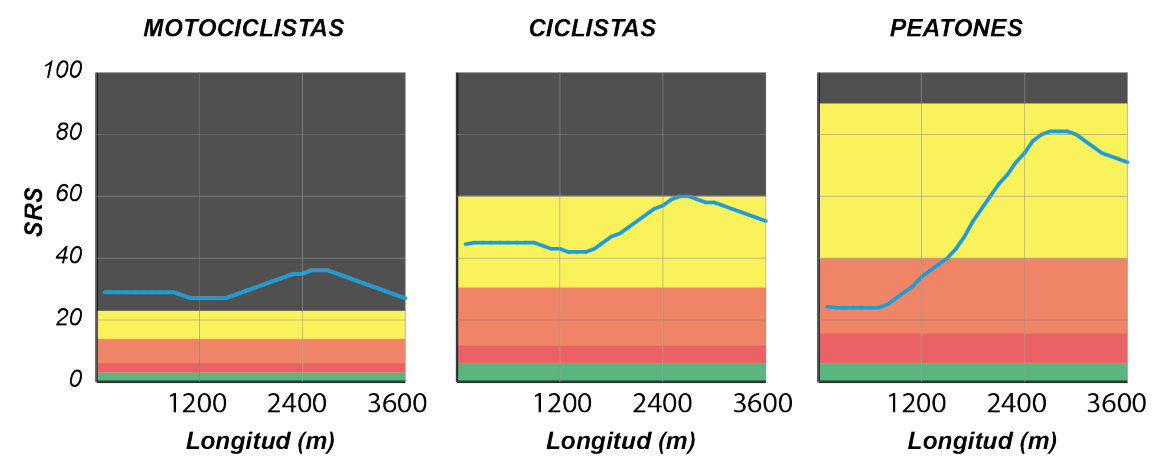

Figura 4. Motociclistas, ciclistas y peatones: valoración de la calificación de estrellas (SRS suavizado) con el número de estrellas (SR) a lo largo del subtramo suburbano de la carretera Loja - Zamora

\subsubsection{Via Loja - Yangana}

En la vía Loja - Yangana sucede algo parecido para ocupante adulto, tal como se ve en la figura 5. En este caso, hay dos subtramos que están cerca de alcanzar las dos estrellas, sin embargo, aún son sitios que representan un peligro para el ocupante adulto en el caso de estar envuelto en algún siniestro vial. 


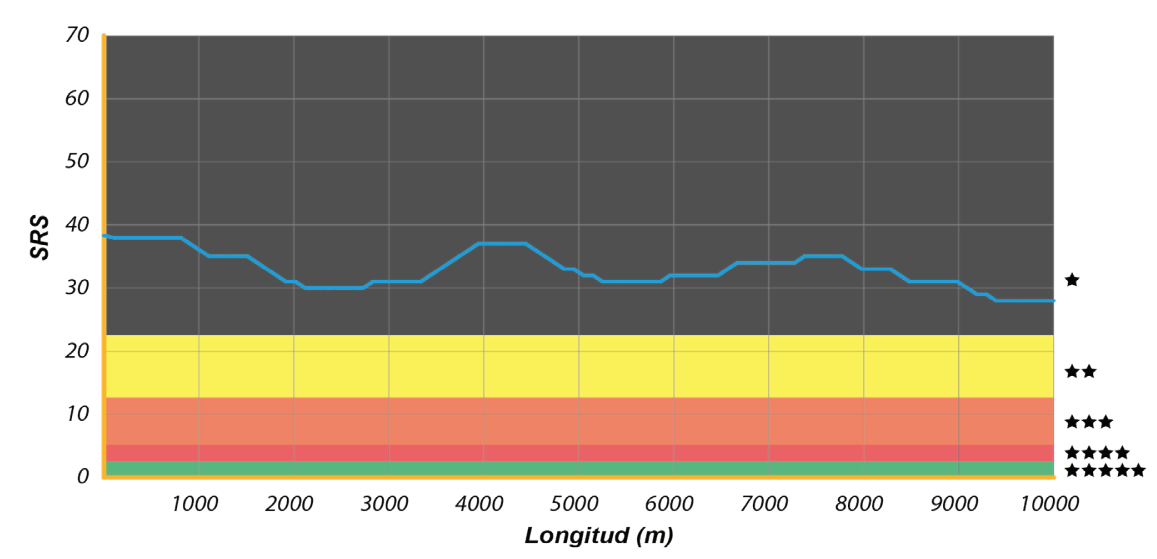

Figura 5. Ocupante de vehículo: valoración de la calificación de estrellas (SRS suavizado) con el número de estrellas (SR) a lo largo de la carretera Loja - Yangana

\subsection{Contramedidas para los sitios en análisis}

Un total de 94 contramedidas se pueden utilizar en el modelo iRAP. Evidentemente su elección dependerá del sitio en sí, de los recursos que se dispongan, de la practicidad de la medida y si su relación costo-beneficio es adecuada. En este caso, se descartaron las medidas costosas como por ejemplo modificaciones en el alineamiento horizontal y perfil; además, de su consecuente interrupción de la operación del tránsito. Luego, se descartaron aquellas que requerían ampliar la sección transversal, tales como aumentar el número de carriles, aumentar el ancho de carril, etc., dado que también son medidas costosas. Finalmente, se eligieron las contramedidas con menor costo e interferencia con el flujo vehicular, como por ejemplo la colocación de guardavías a ambos lados de la carretera, tal como lo muestra en la tabla 3.

En la tabla 3 se puede ver que se ha intervenido en un $39 \%$ en la vía Loja - Zamora y un $42 \%$ en la vía Loja - Yangana. Las valoraciones SRS se graficaron antes y después de las contramedidas para ambas carreteras (ver figuras 6 y 7 ). 
Tabla 3. Contramedidas elegidas para los tramos en estudio

\begin{tabular}{ccccc}
\hline \multirow{2}{*}{$\begin{array}{c}\text { Contramedidas } \\
\text { propuestas }\end{array}$} & \multicolumn{2}{c}{ Vía Loja - Zamora } & \multicolumn{2}{c}{ Vía Loja -Yangana } \\
\cline { 2 - 5 } & $\begin{array}{c}\text { \# de subtramos } \\
\text { intervenidos }\end{array}$ & $\%$ & $\begin{array}{c}\text { \# de subtramos } \\
\text { intervenidos }\end{array}$ & $\%$ \\
\hline $\begin{array}{c}\text { Guardavías - lado } \\
\text { del conductor }\end{array}$ & 58 & 39 & 21 & 21 \\
\hline $\begin{array}{c}\text { Guardavías - lado } \\
\text { del copiloto }\end{array}$ & 58 & 39 & 21 & 21 \\
\hline
\end{tabular}

En la figura 6 se puede ver que alrededor de $12 \mathrm{~km}$, la vía aumentó el número de estrellas 2. Por otro lado, en la carretera Loja - Yangana (figura 7) se disminuyeron los valores de SRS, sin embargo, no fue suficiente para aumentar el número de estrellas.

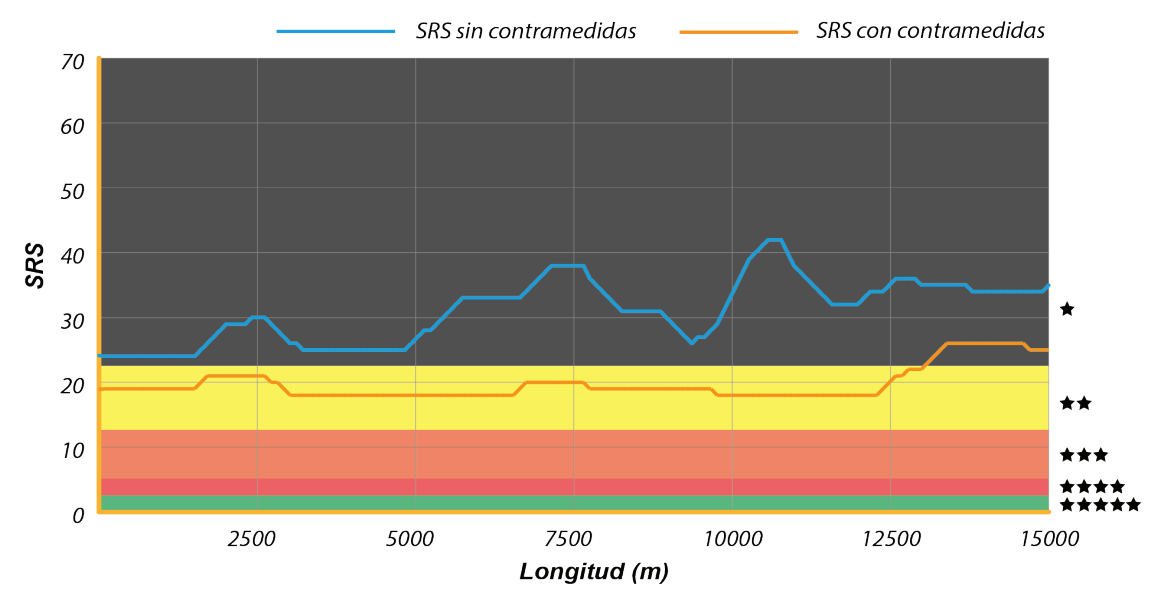

Figura 6. Ocupante de vehículo: comparación entre SRS suavizado sin medidas y el SRS suavizado con medidas a lo largo de la carretera Loja - Zamora

Como se puede ver en ambas figuras, contramedidas relativamente pequeñas pueden ayudar a mejorar la seguridad vial que ofrece un 
subtramo. De todas formas, en estos casos, es necesario utilizar otras contramedidas combinadas con estas, o contramedidas más costosas (modificación de la geometría de la vía) para alcanzar la valoración de 3 estrellas, que es lo que recomienda el modelo iRAP. El objetivo de este apartado no fue alcanzar ese valor, sino solo mostrar que la aplicación de contramedidas sí reduce el valore de SRS y su consecuente posible aumento del SR.

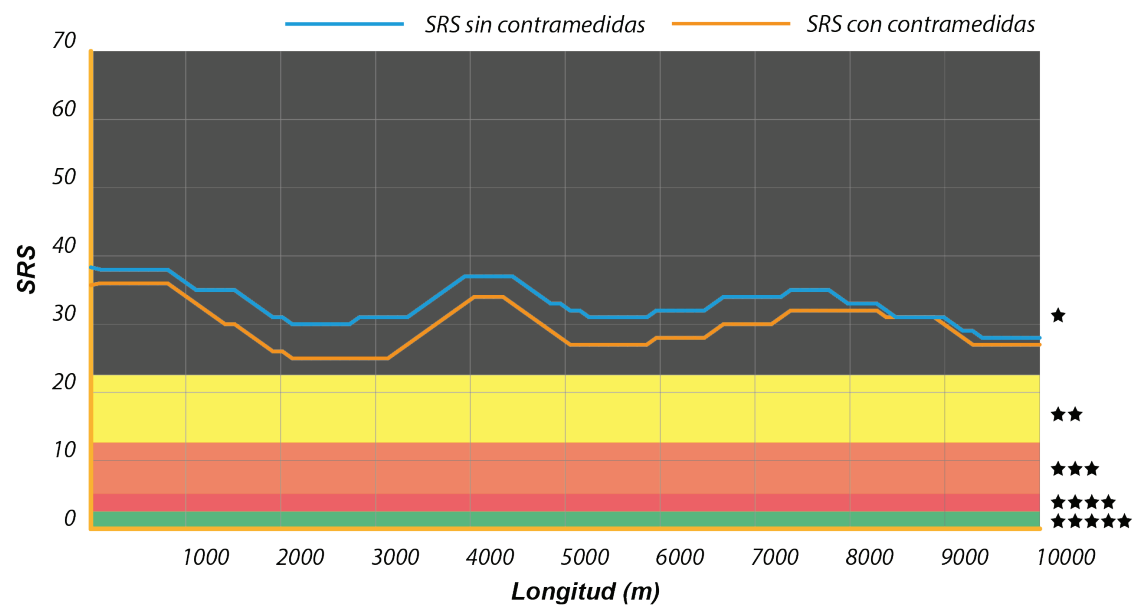

Figura 7. Ocupante de vehículo: comparación entre SRS suavizado sin medidas y el SRS suavizado con medidas a lo largo de la carretera Loja - Yangana

Finalmente, los valores SRS para motociclistas, ciclistas y peatones antes y después de las intervenciones se muestran en la figura 8. En esta se pueden ver que existen una reducción del valor de SRS solo en los motociclistas, dado que las contramedidas de la tabla 3 (guardavías) únicamente afectan a los siniestros con motociclistas. Medidas como construir una ciclovía reducirá el valor de SRS de los ciclistas, mientras que, la construcción de aceras o pasos peatonales reducirán el valor del SRS en peatones. 


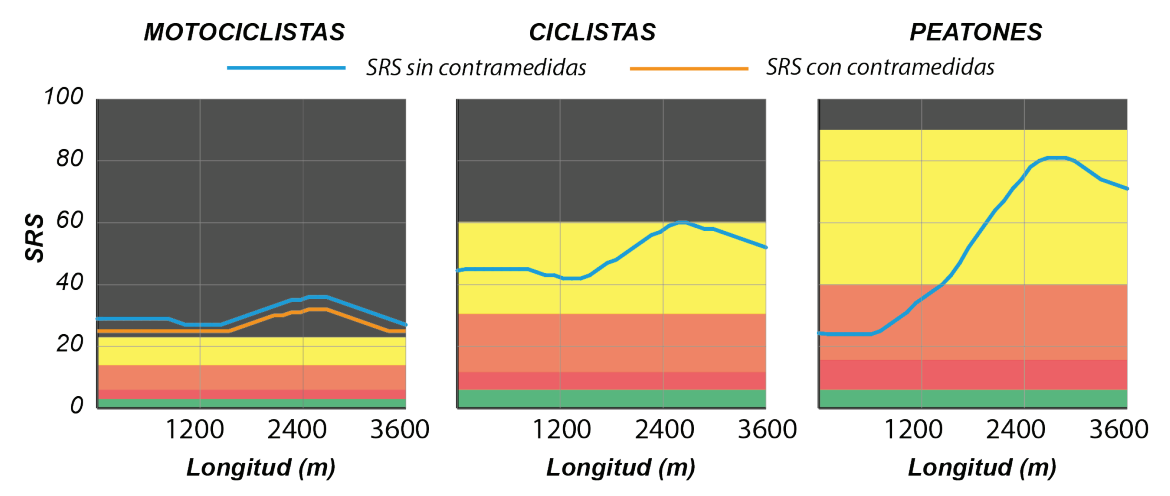

Figura 8. Motociclistas, ciclistas y peatones: comparación entre SRS suavizado sin medidas y el SRS suavizado con medidas a lo largo de la carretera Loja - Yangana

\section{Conclusiones}

Este estudio tuvo por objetivo evaluar el método iRAP y su aplicación a las carreteras de dos carriles del Ecuador. Con este propósito se eligieron dos tramos de dos carreteras que atraviesan topografía montañosa. Los resultados aquí presentados se pueden reproducir fácilmente a otras carreteras del país o en otros países. A partir del análisis de estos resultados, se exponen las principales conclusiones.

Se puede concluir que el modelo iRAP puede ser aplicado fácilmente a este tipo de carreteras, con preparación y la ayuda de programas creados por técnicos o disponibles en línea. Los procedimientos que se deben desarrollar para llegar a una calificación pueden ser aplicados de manera directa. A pesar de obtener un nivel de peligrosidad asociado a la valoración de los sitios, es necesario hacer una calibración local para que el modelo se ajuste a la realidad del país o región de análisis. El modelo iRAP, tal como está planteado, es una herramienta adecuada de planificación, dado que se pueden detectar sitios más riesgosos para los usuarios viales. Sin embargo, a las secciones de $100 \mathrm{~m}$ de longitud, es necesario hacer un recorrido por los lugares en donde se pretende aplicar cierta intervención, y precisar dimensiones acordes a lo que se observe. 
El modelo iRAP puede aportar al debate entre los técnicos sobre la seguridad vial en las carreteras. Esta herramienta también se puede emplear para convencer a los políticos para aplicar una cierta medida, dado que el concepto de número de estrellas es bastante sencillo de entender. También puede utilizarse para que la instrucción formal de conductores sea completada con este modelo, con ello, podrían mejorar su percepción del peligro y su consciencia sobre seguridad vial. Además, el modelo sirve para la prevención, ya que no se debe esperar a que suceda un siniestro para empezar a actuar, si no que se puede hacer con base en esta calificación.

El modelo iRAP tiene una serie de ventajas y desventajas para su aplicación. Como ventajas se tiene que permite planificar, prevenir y extender el conocimiento de la seguridad vial. Mientras que, principalmente, las desventajas son: se debe tener personal capacitado, se debe calibrar los resultados con las condiciones locales, y existe una gran cantidad de variables que medir y/o determinar. Cabe mencionar que hay otros modelos como el IASP, que se reduce el número de variables que se utilizan en el modelo, manteniendo las mismas ventajas que tiene el iRAP. Independiente del modelo que se use, lo importante es mejora la seguridad vial ofrecida por los sitios en las carreteras.

\section{Reconocimientos}

Esta investigación fue financiada por la Universidad Técnica Particular de Loja mediante el proyecto PROY_INV_INGC_2020_2780. Así mismo, un especial agradecimiento a la Secretaría Nacional de Educación Superior, Ciencia, Tecnología e Innovación (SENESCYT) de la República del Ecuador por su apoyo.

\section{Referencias}

Agencia Nacional de Tránsito (2020). Estadísticas sobre Siniestros de Tránsito. Estadísticas de Siniestros de Tránsito - Agencia Nacional de Tránsito Del Ecuador. Disponible en https://www.ant.gob.ec/ index.php/estadisticas

ChinaRAP. (2020). ViDA. Disponible en https://vida.irap.org/es 
García-Ramírez, Y. D., \& Alverca, F. (2019). Calibración de Ecuaciones de Velocidades de Operación en Carreteras Rurales Montañosas de Dos Carriles: Caso de Estudio Ecuatoriano. Revista Politécnica, 43(2), 37-44. Disponible en https://doi.org/10.33333/ rp.vol43n2.1012

Hoque, M. M., Smith, G., Hossain, D. Z., \& Mahmud, S. M. (2010). Improving highway safety in Bangladesh: road improvement and the potential application of iRAP. ARRB Conference, 24th, 2010. Disponible en https://trid.trb.org/view/1096890

iRAP. (2006). Regional RAPs - iRAP. https://irap.org/partnering-tosave-lives/regional-raps/

iRAP. (2013, September 29). iRAP Methodology Fact Sheet \# 9 Star Rating worked example. Disponible en https://irap.org/methodology/

iRAP. (2020). Training - iRAP. Disponible en https://www.irap.org/ training/

Jameel, A. K., \& Evdorides, H. (2016). An investigation for an all-encompassing iRAP road Star Rating index. Functional Pavement Design - Proceedings of the 4th Chinese-European Workshop on Functional Pavement Design, CEW 2016, 1607-1616. Disponible en https://doi.org/10.1201/9781315643274-168

Jurewicz, C., \& Excel, R. (2016). Application of a Crash-predictive Risk Assessment Model to Prioritise Road Safety Investment in Australia. Transportation Research Procedia, 14, 2101-2110. Disponible en https://doi.org/10.1016/j.trpro.2016.05.225

Lawson, S., Barlow, A., Poran, C., Petrosyan, H., \& Sevrovic, M. (2017). Road safety case studies: iRAP road and design assessments and outcomes: A case study from Moldova. Journal of the Australasian College of Road Safety, 28(1). Disponible en https:// search.informit.com.au/documentSummary; $\mathrm{dn}=695101$ 437012748; res=IELNZC

MacAngus, J., Hernandez, H., Milligan, C., Baumgartner, T., Moshiri, M., Patmore, K., Montufar, J., \& Belluz, L. (2011). Risk mapping and a physical-attribute-based star rating system for road safety network screening purposes on canadian prairie region highways. 2011 Conference and Exhibition of the Transportation Association of Canada - Transportation Successes: Let's Build on Them, TAC/ ATC 2011. 
Mcinerney, R., \& Smith, G. (2009). Saving Lives through investment in safer roads: the iRAP partnership. Engineering.

Observatorio de Seguridad Vial. (2020). Sistema Conteo Vehicular. Disponible en https://conteovehicular.kradac.com/utpl/home Organización Mundial de la Salud (2015). Global status report on road safety 2015. Disponible en http://www.who.int/violence_ injury_prevention/road_safety_status/2015/en/

Organización de las Naciones Unidas. (2011). Plan Mundial para el Decenio de Acción para la Seguridad Vial 2011-2020. Disponible en http://www.who.int/roadsafety/decade_of_action/plan/ spanish.pdf

Organización Panamericana de la Salud. (2016). La seguridad vial en la Región de las Américas. Organización Mundial de la Salud. Disponible en https://iris.paho.org/bitstream/handle/10665.2/ 28565/9789275319123-spa.pdf?sequence $=6 \& u a=1$

Zhang, T., Tang, C., Smith, G., \& Wu, L. (2014). Road assessment model and pilot application in China. Discrete Dynamics in Nature and Society, 2014. Disponible en https://doi.org/ $10.1155 / 2014 / 823057$ 